\title{
Produção de palmito de pupunheiras (Bactris gasipaes Kunth) submetidas a diferentes espaçamentos e formas de adubação mineral e orgânica na Amazônia Central
}

\section{Effects of spacing and mineral and organic forms of soil fertilization on the production of peach palm (Bactris gasipaes) heart-of-palm}

\author{
Raimundo Cajueiro LEANDRO ${ }^{1 ; 2}$; Kaoru YUYAMA ${ }^{3}$; Elaine Cristian de Sousa COELHO ${ }^{4}$ \\ ${ }^{1}$ Parte da Tese de Doutorado de Raimundo Cajueiro Leandro, apresentada ao Programa de Pós-graduação em \\ Botânica do Instituto Nacional de Pesquisas da Amazônia-INPA. \\ ${ }^{2}$ Autor para correspondência - Doutor, INPA - Núcleo de Apoio a Pesquisa em Rondônia - Campus da UNIR, \\ BR 364, km 9,5 - 76.801-059 - Porto Velho/RO, e-mail: caju@inpa.gov.br \\ ${ }^{3}$ Pesquisador Titular, Doutor, INPA - Manaus/AM, e-mail: kyuyama@inpa.gov.br \\ ${ }^{4}$ Técnica I/EEFT, INPA - Manaus/AM, e-mail: elaine.coelho@inpa.gov.br
}

Recebido em: 11-02-2013; Aceito em: 14-05-2014

\section{Resumo}

Este trabalho teve como objetivo avaliar a produção de palmito de pupunheiras submetidas a diferentes espaçamentos e formas de adubação mineral e orgânica, na Amazônia Central. O experimento foi conduzido no município de Presidente Figueiredo-AM, Brasil. O delineamento estatístico usado foi o de blocos casualizados com três repetições, segundo esquema fatorial $2 \times 5$, sendo os fatores: dois espaçamentos $(1,0 \times 0,5 \mathrm{~m}$ e $1,0 \times 1,0 \mathrm{~m})$ e adubação (mineral e orgânica). Os tratamentos foram constituídos de: 1) adubação mineral, sob a fórmula 225-90-180 kg ha ${ }^{-1} \mathrm{ano}^{-1}$ de $\mathrm{N}-\mathrm{P}_{2} \mathrm{O}_{5}-\mathrm{K}_{2} \mathrm{O}$; 2) adubação mineral + 2,5 kg de esterco; 3) $5 \mathrm{~kg}$ de torta de filtro; 4) $5 \mathrm{~kg}$ de composto, e 5) adubação mine$\mathrm{ral}+$ composto. A adubação foi na cova, sendo a formulação mineral completa e o esterco aplicados na primeira parcela. As outras três parcelas foram compostas de $\mathrm{N}, \mathrm{K}$ e pelos demais elementos orgânicos. No primeiro ano de produção, não se verificou interação significativa entre adubação e espaçamentos para todas as variáveis avaliadas. Assim, para adubação, as misturas de adubo mineral com o composto ou com esterco de galinha apresentaram maiores valores na produção de estipe e palmito total, enquanto o espaçamento de $1,0 \times 0,5 \mathrm{~m}$ produz mais palmito total, diferindo do segundo espaçamento avaliado. Neste trabalho, não foi constatada diferença significativa entre os fatores comprimento e diâmetro do palmito, tanto nas formas de adubação, quanto nos espaçamentos testados.

Palavras-chave adicionais: densidade de plantio; diâmetro do palmito; população de plantas.

\begin{abstract}
This experiment viewed to evaluate the production of heart-of-palm by peach palms under different spacing and forms of mineral and organic soil fertilization in the Central Amazonian region. The experiment was carried out in the municipality of Presidente Figueiredo, state of Amazonas, Brazil. The experimental design was that of a randomized complete block with three repetitions according to a $2 \times 5$ factorial scheme, the factors being: spacing $(1.0 \times 0.5 \mathrm{~m}$ and $1.0 \times 1.0 \mathrm{~m})$ and mineral and organic fertilization. The treatments consisted of : 1) mineral fertilization whose formula was 225-90$-180 \mathrm{~kg} \mathrm{ha}^{-1}$ year $^{-1}$ of $\mathrm{N}, \mathrm{P} 2 \mathrm{O} 5, \mathrm{~K} 2 \mathrm{O}$; 2) mineral fertilizer $+2.5 \mathrm{~kg}$ of manure; 3$) 5 \mathrm{~kg}$ of filter pie; 4) $5 \mathrm{~kg}$ of compost, and 5) mineral fertilizer + compost. The fertilizers, comprising of manure and the complete mineral fertilizer, were placed in the pit at the first time. The next three times were composed of $\mathrm{N}, \mathrm{K}$, and the other organic elements. In the first year of production, no significant interaction between fertilization and spacing for all the evaluated variables. So, as to soil fertilization, the mixtures of mineral fertilizer and the compost or with chicken manure resulted in higher productions of stem and total heart-of-palm whereas the $1.0 \times 0.5 \mathrm{~m}$ spacing produces more total heart-of-palm. In this work no significant difference between the factors length and diameter of the heart-of-palm were observed.
\end{abstract}

Additional keywords: diameter of the heart-of-palm; planting density; plant population. 


\section{Introdução}

A pupunheira (Bactris gasipaes Kunth) apresenta uma série de vantagens para produção de palmito em relação às outras palmeiras nativas, como o açaí (Euterpe oleracea Martius) e a juçara (Euterpe edulis Martius), explorados de forma extrativista e, às vezes, predatória, portanto apresentando restrições legais e risco de extinção, como no caso do palmito juçara (CHAIMSOHN, 2002).

Quando cultivada em condições adequadas, apresenta rápido crescimento, produzindo palmito de boa qualidade, e o fato de produzir perfilhos confere à espécie o caráter de cultivo perene. Entretanto, talvez por se tratar de uma espécie amazônica, os estudos relacionados à adubação de pupunheiras ainda são poucos. SILVA \& FALCÃO (2002) mencionam que os conhecimentos sobre os aspectos da nutrição mineral da pupunheira para palmito são relativamente escassos e incipientes.

A adubação orgânica em pupunheira para produção de palmito foi pouco estudada, destacando-se trabalhos como de YUYAMA et al. (2002), que constataram que a adição de $2,5 \mathrm{~kg}$ de esterco de galinha na cova proporcionou crescimento similar e superior às plantas, quando comparada com tratamentos usando adubação mineral. Também merece destaque o trabalho de NASCIMENTO et al. (2005), que avaliaram o efeito de esterco bovino na presença e na ausência de NPK para produção de palmito em pupunheira, na localidade de Areia, Paraíba.

Neste contexto, visando ao aproveitamento de resíduos orgânicos gerados na agricultura, à produção agrícola sustentável e à diminuição de custos na agricultura regional, este trabalho teve como objetivo avaliar a produção de palmito, durante o primeiro ano de corte, em pupunheiras adubadas com fertilizantes minerais e orgânicos, em dois diferentes espaçamentos, na Amazônia Central.

\section{Material e métodos}

O experimento foi conduzido na Agropecuária Jayoro Ltda, localizada no município de Presidente Figueiredo, estado do Amazonas, cujas coordenadas geográficas são: latitude: $01^{\circ} 96^{\prime}$ 04 " S e longitude: $60^{\circ} 14^{\prime} 37^{\prime \prime} \mathrm{W}$. O clima é caracterizado como quente e úmido, sendo que o período com maior incidência de chuvas ocorre entre os meses de dezembro e maio. A temperatura média anual é de $31^{\circ} \mathrm{C}$, com máxima de $38 \stackrel{\circ}{\circ}$ e mínima de $20{ }^{\circ} \mathrm{C}$ (NAVA et al., 1998). O plantio foi realizado em área com solo que pertence à classe dos Latossolos Amarelos distróficos típicos, conforme o Sistema Brasileiro de Classificação de Solos (EMBRAPA,
2006).

O delineamento experimental usado foi o de blocos casualizados, com três repetições e 10 plantas na parcela, segundo esquema fatorial $2 \times 5$, sendo os fatores constituídos por: espaçamentos $(1,0 \times 0,5 \mathrm{~m}$ e 1,0 x 1,0 m) e adubação (mineral e orgânica) composta pelos seguintes tratamentos: 1) adubação mineral, sob a fórmula 225-90$-180 \mathrm{kgha}^{-1} \mathrm{ano}^{-1}$ de $\mathrm{N}-\mathrm{P}_{2} \mathrm{O}_{5}-\mathrm{K}_{2} \mathrm{O}$; 2) adubação mineral $\left(\mathrm{N}\right.$ e $\left.\mathrm{K}_{2} \mathrm{O}\right)+2,5 \mathrm{~kg}$ de esterco de galinha por cova; 3) $5 \mathrm{~kg}$ de torta de filtro de cana-de-açúcar por cova; 4) $5 \mathrm{~kg}$ de composto (torta de filtro + casca de guaraná) por cova, e 5) adubação mineral $\left(\mathrm{N}\right.$ e $\left.\mathrm{K}_{2} \mathrm{O}\right)+$ composto $\left(5 \mathrm{~kg} \mathrm{cova}^{-1}\right)$. A formulação mineral completa e o esterco de galinha foram aplicados na primeira adubação. As outras três adubações foram compostas somente por $\mathrm{N}, \mathrm{K}$ e pelos demais elementos orgânicos. Os resíduos orgânicos compostos, torta de filtro e esterco de galinha, tinham a seguinte composição, em g kg ${ }^{-1}: \mathrm{C}=142,14 ; 19,20 ; 90,03$; $\mathrm{N}=11,08 ; 5,39 ; 22,09 ; \mathrm{P}=1,37 ; 3,34 ; 1,34$, e $\mathrm{K}=1,44 ; 11,40 ; 13,40$, respectivamente. O resultado da análise do solo coletado antes da implantação do experimento foi o seguinte: $\mathrm{N}$ $(1,56), C(19,55)$ e matéria orgânica $(33,63) \mathrm{g} \mathrm{kg}^{-1}$; $\mathrm{P}(2,5)$ e K $(0,11) \mathrm{mg} \cdot \mathrm{dm}^{-3}$; Ca $(1,86) ; \mathrm{Mg}(0,76)$, e Al $(0,82) \mathrm{cmol}_{\mathrm{c}} \mathrm{dm}^{-3}$.

As fórmulas para adubação mineral e orgânica foram baseadas em trabalho de FLORES \& YUYAMA (2007) e YUYAMA et al. (2002), que testaram dosagens semelhantes para produção de palmito de pupunha na Amazônia. Em relação aos valores dos elementos da fórmula, HERRERA (1989) constatou que os principais nutrientes exportados no processo de extração do palmito são N e K, e, devido à lenta liberação, o fósforo deve ser parcelado de uma vez (KULCHETSCKI et al., 2001).

O primeiro corte de palmito foi iniciado aos 15 meses, tendo as plantas aproximadamente $1,5 \mathrm{~m}$ de altura, conforme sugerem YUYAMA et al. (2005). Foram colhidos apenas palmitos do estipe principal, desconsiderando-se os perfilhos, uma vez que a metodologia da pesquisa previa análise do primeiro ano de produção. Após o corte do estipe retirou-se do palmito o estipe tenro, conhecido como coração, por situar-se o meristema apical da palmeira nesta região (YUYAMA, 1997).

As varáveis analisadas neste trabalho foram número de estipes e massa de estipe tenro, de palmito e de palmito total (palmito + estipe tenro), além de diâmetro e comprimento do palmito.

Os dados obtidos foram analisados estatisticamente, utilizando-se da análise de variância, pelo teste $F$, e para a comparação de médias, foi utilizado o teste de Tukey, a $5 \%$ de probabilidade. 


\section{Resultados e discussão}

Para todas as variáveis de produção, não foi constatada interação entre os fatores adubação e espaçamentos.

Assim, as maiores produções de palmito, para o fator adubação, foram obtidas com a mistura de adubação mineral e esterco. Entre os espaçamentos estudados, essa produção foi alcançada com o tratamento $\mathrm{E} 1$ de $(1,0 \times 0,5 \mathrm{~m})$. Os dois maiores valores para número de estipes e palmito total não apresentaram diferença estatística entre si, no fator adubação. Entre os espaçamentos adotados, ambas as varáveis diferiram significativamente, prevalecendo 0 espaçamento $1,0 \times 0,5 \mathrm{~m}$ (Tabela 1).

Tabela 1 - Produção de palmito, estipe tenro e palmito total (palmito + estipe tenro), em pupunheiras cultivadas sob diferentes espaçamentos $(E)$ e tipos de adubação $(A)$, no município de Presidente Figueiredo-AM (2008-2009). Production of heart-of-palm, tender stem and total heart-of-palm I (heartof-palm + tender stem), in peach palm grown under different spacing (E) and forms of mineral and organic fertilization (A) in Presidente Figueiredo-AM (2008-2009).

\begin{tabular}{|c|c|c|c|c|}
\hline Tratamentos & $\begin{array}{l}{ }^{(a)} \text { Número de } \\
\text { Estipes }(\times 1000)\end{array}$ & ${ }^{(\mathrm{a})}$ Palmito & $\begin{array}{l}{ }^{(\mathrm{a})} \text { Estipe tenro } \\
\left(\mathrm{kg} \mathrm{ha}^{-1}\right)\end{array}$ & ${ }^{(\mathrm{a})}$ Palmito Total \\
\hline \multicolumn{5}{|l|}{ Adubação (A) } \\
\hline A1-Mineral & $13,33 \mathrm{~B}$ & $2,559 \mathrm{AB}$ & $2,749 \mathrm{~B}$ & $5,304 \mathrm{~B}$ \\
\hline A2-Mineral+Esterco & $24,50 \mathrm{~A}$ & $4,404 \mathrm{~A}$ & $4,946 \mathrm{~A}$ & $9,351 \mathrm{~A}$ \\
\hline A3-Torta de filtro & $12,00 \mathrm{~B}$ & $1,993 \mathrm{~B}$ & $2,383 \mathrm{~B}$ & $4,376 \mathrm{~B}$ \\
\hline A4-Composto & $11,17 \mathrm{~B}$ & $2,112 \mathrm{~B}$ & $2,287 \mathrm{~B}$ & $4,399 \mathrm{~B}$ \\
\hline A5-Mineral+Composto & $14,83 \mathrm{AB}$ & $2,570 \mathrm{AB}$ & $2,911 \mathrm{AB}$ & $5,481 \mathrm{AB}$ \\
\hline \multicolumn{5}{|l|}{ Espaçamento (E) } \\
\hline $\mathrm{E} 1-(1,0 \times 0,5 \mathrm{~m})$ & $19,87 \mathrm{a}$ & $3,531 \mathrm{a}$ & $3,870 \mathrm{a}$ & $7,401 \mathrm{a}$ \\
\hline E2 - $(1,0 \times 1,0 \mathrm{~m})$ & $10,47 b$ & $1,924 \mathrm{~b}$ & $2,241 \mathrm{~b}$ & $4,164 \mathrm{~b}$ \\
\hline C.V. (\%) & 39,18 & 39,43 & 39,71 & 38,96 \\
\hline
\end{tabular}

${ }^{\text {(a) }}$ Médias dos tratamentos de adubação seguidas de mesma letra maiúscula na coluna não diferem estatisticamente entre si, pelo teste de Tukey $(p<0,05)$, e médias dos tratamentos de espaçamentos seguidas de mesma letra minúscula na coluna não diferem estatisticamente entre si, pelo teste de Tukey $(p<0,05)$.

BEZERRA \& YUYAMA (2006b), testando espaçamentos de $2,0 \times 1,0 \mathrm{~m}, 2,0 \times 0,5 \mathrm{~m}, 1,5 \times$ $1,0 \mathrm{~m}$ e 1,0 x 1,0 m e 1,0 x 0,5 m, com adubação mineral com 225-90-180 kg.ha ${ }^{-1}$ de N-P-K e orgânica, com $2 \mathrm{~kg}$ de esterco de galinha cova ${ }^{-1}$ em pupunheiras para palmito, no município de Manaus-AM, verificaram que o espaçamento de $1,0 \times 0,5 \mathrm{~m}$ promoveu maior produção em número de estipes. Esse resultado está de acordo com os dados obtidos nesta pesquisa.

FLORES \& YUYAMA (2007), analisando adubação orgânica e mineral em pupunheiras com e sem espinhos, plantadas no Estado do Amazonas, sob o espaçamento de 2,0 x 1,0 m, mencionam não haver diferença significativa entre os tratamentos que receberam adubação. Os autores ainda relatam que, aos 12 meses de idade, a produção obtida foi de 5.000 estipes por $\mathrm{ha}^{-1}$, diante de plantas adubadas com tratamento contendo adubo mineral parcelado $\left(75 \mathrm{~kg} \mathrm{ha}{ }^{1}\right.$ de $\mathrm{N}, 90 \mathrm{~kg} \mathrm{ha}{ }^{-1}$ de $\mathrm{P}_{2} \mathrm{O}_{5}$ e $60 \mathrm{~kg} \cdot \mathrm{ha}^{-1}$ de $\left.\mathrm{K}_{2} \mathrm{O}\right)$ e 4.500 estipes por hectare, em tratamentos com adubação de $50 \%$ de esterco (12,5 ton.ha $\left.{ }^{-1}\right)+$ adubo mineral parcelado e adubo mineral em cobertura, concordando, parcialmente, com este trabalho. Entretanto, YUYAMA et al. (2005), estudando cultivo de pupunheiras para palmito no Amazonas, concluíram que adubação mineral similar à utilizada neste trabalho e densidade de 10.000 plantas ha $^{-1}$ geraram a produção de 4.000 estipes ha ${ }^{-1}$ ano $^{-1}$. Os dados apresentados no trabalho de YUYAMA et al. (2005) evidenciam a necessidade da adição de adubo orgânico no cultivo de pupunheira para palmito.

Em trabalho conduzido no Estado do Paraná, NEVES et al. (2006) mencionam que, em corte realizado aos 24 meses, a produção obtida foi de $4.066 \mathrm{~kg} \mathrm{ha}^{-1} \mathrm{ano}^{-1}$ de creme de palmito, sendo 8.000 a densidade de plantas por hectare.

Observando os valores da quantidade de estipes coletados (Tabela 1), com a maior produção obtida com adubação mineral + esterco, confirmamos a necessidade no uso do adubo mineral associado à adubação orgânica, em pupunheira, para a produção de palmito, como destacam NASCIMENTO et al. (2005).

A Tabela 1 exibe ainda o resultado para produção de palmito total, que consiste na soma do estipe tenro mais o palmito, no primeiro ano. Nos caracteres número de estipe, número de palmito, estipe tenro e palmito total, os dois maiores valores foram verificados nos tratamentos A2 (mineral + esterco) e A5 (mineral + composto), que não diferiram significativamente. $O$ resultado deste estudo corrobora o obtido por FLORES \& YUYAMA (2007) que, pesquisando adubações orgânica e mineral na Amazônia Central, concluíram que a adubação com esterco em cobertura e adubação orgânica e mineral proporcionaram maior rendimento de palmito e estipe tenro.

Também na Amazônia, BEZERRA \& YUYAMA (2006a) comprovaram que o espaça- 
mento $1,0 \times 0,5 \mathrm{~m}$ produziu maior número de palmitos por hectare, diferindo de outros espaçamentos analisados $(2,0 \times 1,0 \mathrm{~m}$ e 1,5 x 0,5 m). Analisando produção de palmito de pupunheira na Amazônia, plantada sob espaçamento de 2,0 x 1,0 m, FLORES \& YUYAMA (2007) verificaram que os tratamentos com adubação orgânica (esterco em cobertura, com $25 \mathrm{t} \mathrm{ha}^{-1}$ ) e orgânica + mineral $\left(50 \%\right.$ de esterco na cova $\left(12,5 \mathrm{tha}^{-1}\right)+$ $50 \%$ de adubo mineral $(112,5 \mathrm{~kg}$ de N, $45 \mathrm{~kg}$ de $\mathrm{P}_{2} \mathrm{O}_{5}$ e $90 \mathrm{~kg} \mathrm{ha}^{-1}$ de $\mathrm{K}_{2} \mathrm{O}$ ) proporcionaram o maior rendimento de palmito.

Nas variáveis diâmetro e comprimento, os palmitos coletados no estipe principal não apresentaram diferença significativa em nenhum dos tratamentos, tanto no fator adubação quanto nos espaçamentos pesquisados (Tabela 2). Entretanto, o diâmetro médio do palmito, no primeiro ano de produção, foi maior que o obtido por FLORES (2003), em ensaio de adubações orgânica e mineral no Estado do Amazonas, utilizando pupunheiras com espaçamento de $2,0 \times 1,0 \mathrm{~m}$. No referido trabalho, o autor encontrou diâmetro de até $29,20 \mathrm{~mm}$, no tratamento com adubo mineral parcelado. Esses valores são similares aos alcançados nesta pesquisa, onde também não houve diferença entre os níveis de adubação nem nos diferentes espaçamentos pesquisados. Todavia, em relação aos espaçamentos, o diâmetro do palmito apresenta valores menores que os de FLORES (2003), provavelmente, por se tratar de plantio mais adensado, onde a concorrência por luz leva a planta a crescer mais verticalmente. FLORI et al. (2001), pesquisando o rendimento do palmito de pupunha em função da densidade de plantio, diâmetro de corte e manejo dos perfilhos, na Bahia, obtiveram palmitos com 33,40 e $34,11 \mathrm{~mm}$ de diâmetro, com adubação mineral e espaçamentos de $2,0 \times 1,0 \mathrm{~m}$ e $2,0 \times 1,5 \mathrm{~m}$, respectivamente. Os autores destacam que o espaçamento evidencia efeitos significativos no diâmetro do palmito.

Tabela 2 - Diâmetro e comprimento dos palmitos colhidos no primeiro ano de produção em pupunheiras, submetidas a diferentes espaçamentos (E) e adubações (A), em Presidente Figueiredo-AM (20082009). Diameter and length (cm) of the heart-of-palm, harvested in the first year of production in peach palms, under different spacing $(E)$ and fertilizations (A), in Presidente Figueiredo-AM (2008-2009).

\begin{tabular}{|c|c|c|}
\hline \multirow{2}{*}{ Tratamentos } & (a)Diâmetro & ${ }^{(\mathrm{a})}$ Comprimento \\
\hline & \multicolumn{2}{|c|}{---------------- (cm) --------------- } \\
\hline Adubação (A) & & \\
\hline A1-Mineral & $2,82 \mathrm{~A}$ & $34,06 \mathrm{~A}$ \\
\hline A2-Mineral+Esterco & $2,77 \mathrm{~A}$ & $34,94 \mathrm{~A}$ \\
\hline A3-Torta de filtro & $2,75 \mathrm{~A}$ & $31,14 \mathrm{~A}$ \\
\hline A4-Composto & $2,92 \mathrm{~A}$ & $31,26 \mathrm{~A}$ \\
\hline A5-Mineral+Composto & $2,65 \mathrm{~A}$ & $34,42 \mathrm{~A}$ \\
\hline Espaçamento (E) & & \\
\hline $\mathrm{E} 1-(1.0 \times 0,5 \mathrm{~m})$ & $2,78 \mathrm{a}$ & $33,15 a$ \\
\hline E2 - $(1,0 \times 1,0 \mathrm{~m})$ & $2,78 \mathrm{a}$ & $33,18 \mathrm{a}$ \\
\hline C.V. (\%) & 5,77 & 8,37 \\
\hline
\end{tabular}

MORAES NETO et al. (2000) afirmam que várias características constituem parâmetros para avaliar as respostas de crescimento de plantas à intensidade luminosa. Dentre essas, a de uso mais frequente é a altura da planta, visto que a capacidade em crescer rapidamente quando sombreadas é um mecanismo de aclimatação das plantas, compreendendo uma valiosa estratégia para escapar do sombreamento. Nesse sentido, KULCHETSCKI et al. (2001) garantem que plantios mais adensados produzem palmitos mais finos, indicando, assim, a necessidade de manejo das plantas, caso o mercado exija palmito com maior diâmetro.

$\mathrm{Na}$ estimativa apresentada para os dados médios de comprimento do palmito da planta principal, FLORES (2003) foi verificado que, nos tratamentos que receberam adubação, não foram constatadas diferenças significativas entre os níveis estudados, tendo registrado 32,33 e $31,99 \mathrm{~cm}$ de comprimento, nos tratamentos com adubação de esterco na cova e esterco em cobertura, respectivamente. Contudo, em pesquisa com espaçamento de 2,0 x 1,0 m e adubação mineral, YUYAMA et al. (2005) obtiveram palmitos com diâmetro médio de $2,4 \mathrm{~cm}$ e comprimento de $21,5 \mathrm{~cm}$. Os mesmos autores, com adubação semelhante à deste trabalho, produziram palmitos com $3,4 \mathrm{~cm}$ e $29 \mathrm{~cm}$, para diâmetro e comprimento, respectivamente. Os dados mostrados nos diferentes tipos de adubação indicam que a interação entre adubo mineral e orgânico pode resultar em melhores médias de comprimento de palmito (YUYAMA, 1997).

No Estado do Paraná, OLIVEIRA et al. (2001), analisando fontes e doses de matéria orgânica, incluindo esterco de galinha, no crescimento de pupunheira, também concluíram que 
esterco bovino conferiu maior altura à pupunheira, consequentemente ao comprimento do palmito, sendo considerado o mais eficiente.

Entre as densidades estudas, os números encontrados por YUYAMA et al. (2005), com espaçamento de 2,0 x 1,0 m, são menores que os alcançados neste estudo (Tabela 2). Neste sentido, analisando o efeito de diferentes espaçamentos no crescimento de pupunheira na Amazônia Central, BEZERRA \& YUYAMA (2006a) concluíram que a alta densidade (20.000 plantas ha ${ }^{-1}$ ) tende a diminuir o diâmetro do palmito, o que não significa, necessariamente, menor rendimento de palmito por hectare. Ademais, a densidade (espaçamento) de plantas pode ser manejada conforme a produtividade desejada ou a exigência de mercado, sendo o palmito fino (1,5 a $3 \mathrm{~cm}$ de diâmetro) mais adequado à exportação (YUYAMA et al., 2005).

Utilizando a mesma fórmula de adubação mineral e $1,5 \mathrm{~kg}$ de esterco na cova, FREIRE (2011) estudou o ponto ótimo para colheita de palmito, em densidade de 20.000 plantas ha ${ }^{-1}$, em Manaus-AM, obtendo palmito com média de comprimento de $31,88 \mathrm{~cm}$ e de diâmetro de $2,42 \mathrm{~cm}$, em plantas com altura média de 1,87 $\mathrm{m}$. Esses dados confirmam a eficácia da interação entre adubos mineral e orgânico no cultivo de pupunheira para palmito na Amazônia.

A população Yurimaguas, com a qual trabalhamos neste ensaio, apresenta material promissor para programas de melhoramento, pois algumas plantas produzem palmito com mais de $45 \mathrm{~cm}$ de comprimento, o que possibilita maior rendimento por estipe (YUYAMA et al., 2002).

\section{Conclusões}

No primeiro ano de produção de palmito de pupunheiras na Amazônia Central, conclui-se que:

Entre os tipos de adubação testados, a mistura de esterco de galinha com adubo mineral mostra-se mais vantajosa do que a adubação mineral sozinha ou misturada com os demais adubos orgânicos testados, porque, embora não produza palmito de maior tamanho em diâmetro e em comprimento, sua aplicação aumenta o número de estipes e a produtividade de estipes e de palmitos.

O espaçamento de $1,0 \times 0,5 \quad \mathrm{~m}$ (20.000 plantas ha ${ }^{-1}$ ) produz mais palmito total, no primeiro ano de colheita, mas não resulta em palmitos com maiores diâmetro e comprimento do que os produzidos pelas plantas no espaçamento $1,0 \times 1,0 \mathrm{~m}\left(10.000\right.$ plantas ha $\left.{ }^{-1}\right)$.

\section{Agradecimentos}

Ao CNPq, pelo apoio financeiro, a Usina Jayoro, pela cessão da área experimental e dos funcionários para instalação e manutenção do experimento.

\section{Referências}

BEZERRA, R. L.; YUYAMA, K. Efeito de espaçamento no crescimento de pupunheira na Amazônia Central. In: CONGRESSO BRASILEIRO DE OLERICULTURA, 46., 2006a, Goiânia. Anais...Natal: Sociedade Brasileira de Entomologia, 2010.

BEZERRA, R. L.; YUYAMA, K. Efeito de espaçamento e adubação na produção de palmito de pupunheira na Amazônia Central. In: CONGRESSO BRASILEIRO DE OLERICULTURA, 46, 2006b, Goiânia. Horticultura Brasileira, suplemento. Goiânia, GO: ABH, v.24, p.21722175, 2006b

CHAIMSOHN, F. P. Desenvolvimento de pupunha (Bactris gasipaes Kunth) cultivada em diferentes regiões do Paraná. 1-54. In: GALDINO, N. O.; CLEMENTE, E. 2008. Palmito de pupunha (Bactris gasipaes Kunth) composição mineral e cinética de enzimas oxidativas. Ciencia e Tecnologia de Alimentos, Campinas, v.28, n.3, p.540-544, 2002.

EMBRAPA. Centro Nacional de Pesquisa de Solos. Sistema brasileiro de classificação de solos. 2.ed. Rio de Janeiro: Embrapa Solos, 2006. 306p.

FLORES, W. B. C. Padrão de desenvolvimento da pupunheira (Bactris gasipaes Kunth) com e sem espinhos, em diferentes formas de adubação para a produção de palmito na Amazônia Central. 2003. 118f. Tese (Doutorado em Botânica) - Instituto Nacional de Pesquisas da Amazônia, Manaus, 2003.

FLORES, W. B. C.; YUYAMA, K. Adubação orgânica e mineral para a produção de palmito da pupunheira na Amazônia Central. Acta Amazonica, Manaus, v.37, n.4, p.483-490, 2007.

FLORI, J. E.; RESENDE, G. M.; DRUMOND, M. A. Rendimento do palmito de pupunha em função da densidade de plantio, diâmetro de corte e manejo dos perfilhos, no Vale do São Francisco. Horticultura Brasileira, Brasília, v.19, n 2: p.140$-143,2001$.

FREIRE, D. C. B. Determinação do ponto ótimo para extração do palmito de pupunheira, análise nutricional e rendimento em função dos períodos pluviométricos na Amazônia Central. 2011. 67f. Dissertação (Mestrado em Agricultura no Trópico Úmido), - Instituto Nacional de Pesquisas da Amazônia, Manaus, 2003.

HERRERA, B. W. Fertilización del pejibaye para palmito. Boletin informativo "Pejibaye (Guilielma)", Costa Rica, v.1, n.2: p.4-10, 1989. 
KULCHETSCKI, L; $\quad$ CHAIMSOHN, F. P.; GARDINGO, J. R. (Ed.). Palmito Pupunha (Bactris gasipaes Kunth): a espécie, cultura, manejo agronômico, usos e processamentos. Ponta Grossa: Editora UEPG, 2001. 148 p.

NASCIMENTO, J. T.; OLIVEIRA, A. P.; SOUZA, A. P.; SILVA, I. F.; ALVES, A. U. Rendimento de palmito de pupunheira em função da aplicação de esterco bovino e adubação química. Horticultura Brasileira, Brasília, v.23, n.1, p.19-21, 2005.

NAVA, D. B.; MONTEIRO, E. A.; CORREIA, M. C.; ARAÚJO, M. R.; SAMPAIO, R. R. L.; CAMPOS, G. S. Companhia de Pesquisa de Recursos Minerais - CPRM. Sócio - Economia do Município de Presidente Figueiredo, Amazonas, p.63, 1998.

NEVES, E. J. M.; SANTOS, A. F.; MARTINS, E. G.; KALIL FILHO, A. N. 2006. Época de corte da pupunheira (Bactris gasipaes Kunth.) para a produção de palmito em pequenas propriedades no litoral do Paraná. Colombo-PR: Embrapa Florestas, 2006. 3p. (Comunicado Técnico, 162).
OLIVEIRA, A. P.; ALVES, A. U; CANDEIA, B. L.; BENVINDO, J. M. S.; BARBOSA, L. J. N.; SILVA, J. V.. 2001. Crescimento da pupunheira em função de fontes e doses de matéria orgânica. In: 41 CONGRESSO BRASILEIRO DE OLERICULTURA, 41., 2001, Brasília. Palestras... CD-ROM.

SILVA, J. R. A.; FALCÃO, N. P. S. Caracterização de sintomas de carências nutricionais em mudas de pupunheira cultivadas em solução nutritiva. Acta Amazonica, Manaus, v.32, n.4, p.529-539. 2002.

YUYAMA, K.; CHÁVEZ F., W. B.; CLEMENT, C. R. Pupunheira. In: BRUCKNER, C. H. (Ed.) Melhoramento de fruteiras tropicais. Viçosa: UFV, 2002, p.411-422.

YUYAMA, K.; FLORES, W. B. C.; PEREIRA, B. G.; SILVA, I. A. Efeito da densidade de plantas e da adubação NPK na produção inicial de palmito de pupunheira. Revista Brasileira de Ciência do Solo, Viçosa, MG, v.29, p.373-378, 2005. 